\title{
菲啶类化合物的合成新方法
}

\author{
马丽芳吕文贤黄丹凤* 牛腾苏瀛鹏 \\ 王克虎* 胡雨来 \\ (西北师范大学化学化工学院 兰州 730070)
}

\begin{abstract}
摘要 探索了 5 -甲氧基菲啶-6(5H)-酮化合物与芳基格氏试剂的反应，得到了菲啶类化合物. 该方法反应条件温和，操 作简便, 产率较好, 提供了一种合成菲啶衍生物的新方法. 但是, 当烷基溴化镁与 5 -甲氧基菲啶- $6(5 H)$-酮化合物反应 时, 只得到了菲啶-6(5H)-酮类化合物.
\end{abstract}

关键词＼cjkstart菲啶; 5-甲氧基菲啶-6(5H)-酮; Weinreb 酰胺; 格氏试剂; 合成

\section{A New Method for the Synthesis of Phenanthridine Compounds}

\author{
Ma, Lifang \\ Lü, Wenxian \\ Huang, Danfeng* Niu, Teng \\ $\mathrm{Su}$, Yingpeng \\ Wang, Kehu* Hu, Yulai \\ (College of Chemistry and Chemical Engineering, Northwest Normal University, Lanzhou 730070)
}

\begin{abstract}
Methoxyphenanthridin-6(5H)-ones reacted with aryl Grignard reagents to afford phenanthridine compounds. The method has the advantages of mild reaction conditions with good yields and provides a new synthetic procedure for the phenanthridine derivatives. However, the products were phenanthridin-6(5H)-ones when alkyl magnesium bromide reacted with 5-methoxyphenanthridin-6(5H)-ones.
\end{abstract}

Keywords phenanthridines; 5-methoxyphenanthridin-6(5H)-ones; Weinreb amides; Grignard reagents; synthesis

菲啶是一类重要的杂环化合物. 由于这类化合物具 有潜在的生物活性和光电特性, 其结构单元广泛存在于 一些天然产物 ${ }^{[1]}$ 、药物 ${ }^{[2]}$ 和功能材料分子中 ${ }^{[3]}$. 例如, 白 屈菜赤碱(Chelerythrine)和两面针碱(Nitidine)及花椒宁 碱(Fagaronine)都属于菲啶生物碱 ${ }^{[4]}$, 前者具有抗细胞毒 素、抗菌等活性, 后二者具有抗癌活性. 因此, 探索菲啶 化合物的高效选择性合成方法在医药化学和材料科学 领域中具有重要意义. 在已报道的菲啶化合物的各种合 成方法中, 邻取代的二芳基化合物的分子内的环化为主 要合成方法. 目前, 合成菲啶衍生物的分子内环化方法 主要有: 自由基环合法 ${ }^{[5]}$ 、苯炔法 ${ }^{[6]}$ 、多组分一锅串联 法 ${ }^{[6 \mathrm{a}, 6 \mathrm{~b}, 7]}$ 、光化学法 ${ }^{[8]}$ 、微波促进法 ${ }^{[9]}$ 和过渡金属催化 法 ${ }^{[10]}$ 等. 然而，在这些方法中，仍存在一定的局限性： 如反应原料不易获得, 反应条件苛刻, 步骤较多, 产率 不高, 不具有普遍应用性等. 因此, 探索高效、简便、条 件温和的合成菲啶及其衍生物的新方法仍具有重要的
意义.

另一方面, $N$-甲基- $N$-甲氧基酰胺(Weinreb 酰胺)是 一类十分重要的酰基化试剂 ${ }^{[11]}$. 这类酰胺与其它酰胺 不同, 它与金属有机试剂(如 Grignard 试剂)反应时生成 的产物是酮而不是醇. 目前, Weinreb 酰胺已被广泛应 用于许多天然产物及药物分子的全合成中 ${ }^{[12]}$. 我们小 组 ${ }^{[13]}$ 对 Weinreb 酰胺的合成及应用也展开了一系列研究 工作. 为了进一步探索 $N$-甲基- $N$-甲氧基酰胺的性质, 扩大它在有机合成中的应用, 我们选用与 Weinreb 酰胺 结构类似的 5-甲氧基菲啶-6(5H)-酮为原料，使它与格氏 试剂反应，希望得到相应的酮类化合物. 但是我们发现: 5-甲氧基菲啶-6(5H)-酮化合物与芳基格氏试剂反应后 得到的产物主要是 6-取代的菲啶化合物, 而不是所希望 的酮类化合物(Scheme 1). 由于菲啶化合物是一些天然 产物和药物合成的重要中间体, 因此本文报道一种由 5甲氧基菲啶-6(5H)-酮化合物与格氏试剂合成菲啶化合

\footnotetext{
*E-mail: huangdf@nwnu.edu.cn; wangkh@nwnu.edu.cn

Received October 15, 2013; revised December 10, 213; published online January 15, 2014.

Project supported by the National Natural Science Foundation of China (No. 21062016).

国家自然科学基金(No. 21062016)资助项目.
} 
物的新方法.

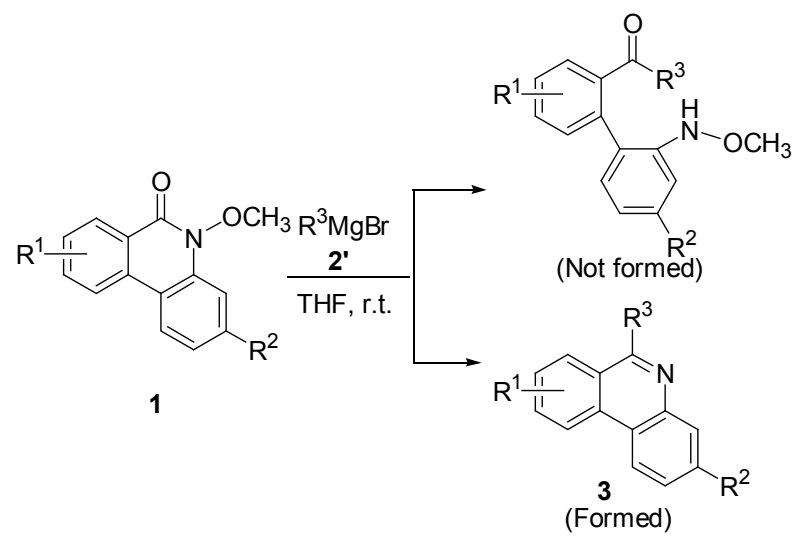

Scheme 1

\section{1 结果与讨论}

\section{1 反应条件的优化}

我们首先将 5-甲氧基菲啶-6(5H)-酮(1a) (1 equiv.) 和溴苯(2a) (1.2 equiv.)制成的苯基溴化镁试剂, 在 THF 溶剂中室温摚拌. TLC 监测反应结束后, 得到了菲啶化 合物 3a (14\%产率)和化合物 4a (57\%产率) (Scheme 2). 然后我们以化合物 $\mathbf{3 a}$ 的合成为目标, 对反应的物料比、 溶剂和反应温度等条件进行了优化, 结果列于表 1 .

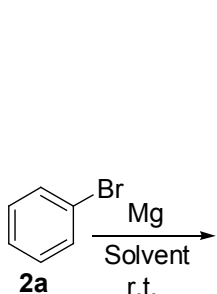<smiles>Cc1ccc2c(c1)nc(-c1ccccc1)c1ccccc12</smiles>

3a
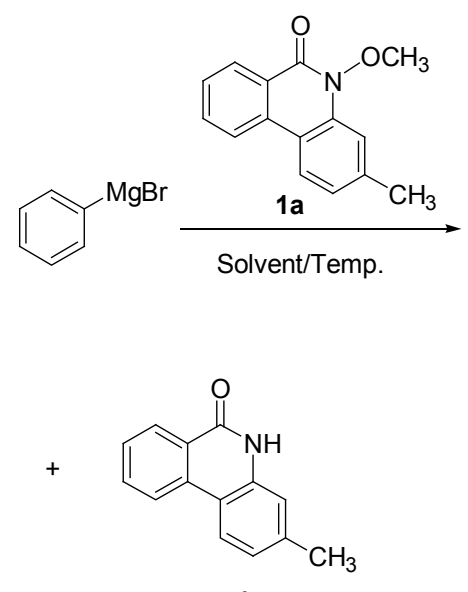

$4 a$

Scheme 2

由表 1 中 Entries $1 \sim 8$ 的数据可以看出, 随着 5-甲 氧基菲啶-6(5H)-酮(1a)和溴苯(2a) 的物质的量比增大, 化合物 3a 的产率逐渐升高, 化合物 $4 \mathrm{a}$ 的产率不断下降. 当 $1 \mathrm{a}$ 和 $2 \mathrm{a}$ 的物质的量比增大到 $1: 5$ 时, 化合物 $3 \mathrm{a}$ 的 产率可达到 $75 \%$, TLC 显示有痕量的 $4 \mathbf{a}$ 生成. 继续增加 $\mathbf{2 a}$ 的量, 化合物 $\mathbf{3 a}$ 的产率没有明显升高.

由表 1 中 Entries 9 12 的数据可以看出, 在乙醚和 1,4-二氧六环中, 由于反应底物的溶解性不好, 化合物 $3 \mathbf{a}$ 的产率不高甚至反应不能发生; 当用四氢呋喃(THF)
表 1 条件优化的反应结果 ${ }^{a}$

Table 1 The results of optimization of the reaction conditions

\begin{tabular}{|c|c|c|c|c|c|}
\hline \multirow{2}{*}{ Entry } & \multirow{2}{*}{ Solvent } & \multirow{2}{*}{$\begin{array}{c}\text { Molar ratio } \\
\text { of } \mathbf{1 a} / \mathbf{2 a}\end{array}$} & \multirow{2}{*}{ Temp. $/{ }^{\circ} \mathrm{C}$} & \multicolumn{2}{|c|}{ Yield $^{b} / \%$} \\
\hline & & & & $3 \mathbf{a}$ & $4 a$ \\
\hline 1 & THF & $1: 1.2$ & r.t. & 14 & 57 \\
\hline 2 & THF & $1: 2.0$ & r.t. & 39 & 30 \\
\hline 3 & THF & $1: 2.5$ & r.t. & 52 & 15 \\
\hline 4 & THF & $1: 3.0$ & r.t. & 56 & 13 \\
\hline 5 & THF & $1: 3.5$ & r.t. & 65 & 13 \\
\hline 6 & THF & $1: 4.0$ & r.t. & 69 & 11 \\
\hline 7 & THF & $1: 5.0$ & r.t. & 75 & Trace \\
\hline 8 & THF & $1: 6.0$ & r.t. & 73 & Trace \\
\hline 9 & $\mathrm{Et}_{2} \mathrm{O}$ & $1: 5.0$ & r.t. & 20 & Trace \\
\hline 10 & 1,4-Dioxane & $1: 5.0$ & r.t. & - & - \\
\hline 11 & $\mathrm{Et}_{2} \mathrm{O} / \mathrm{THF}$ & $1: 5.0$ & r.t. & 32 & Trace \\
\hline 12 & 1,4-Dioxane/THF & $1: 5.0$ & r.t. & 40 & 5 \\
\hline 13 & THF & $1: 5.0$ & 35 & 60 & Trace \\
\hline 14 & THF & $1: 5.0$ & 45 & 59 & Trace \\
\hline 15 & THF & $1: 5.0$ & 60 & 36 & 24 \\
\hline 16 & THF & $1: 5.0$ & 0 & trace & Trace \\
\hline
\end{tabular}

溶解化合物 2a, 再分别加到乙醚和 1,4-二氧六环制成的 格氏试剂中，反应活性也不是很好. 另外，反应温度对 此反应也有一定影响(Entries 13 16). 当反应温度升高, 化合物 $3 \mathbf{a}$ 的产率下降, 当反应温度降到 $0{ }^{\circ} \mathrm{C}$ 时, 没有 得到化合物 $\mathbf{3 a}$.

综上, 我们确定生成 $\mathbf{3 a}$ 的最优反应条件为: 反应温 度为室温, 时间 $12 \mathrm{~h}$, 溶剂为四氢呋喃, $1 \mathrm{a}$ 和溴苯的物 质的量比为 $1: 5$.

\section{2 反应底物拓展}

在上述优化反应条件下, 我们对此反应的底物进行 了拓展(Eq. 1), 反应结果列于表 2 .

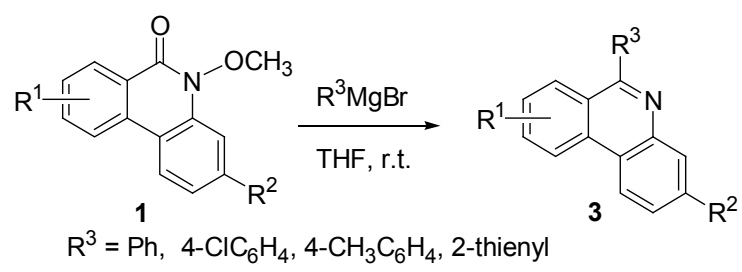

从表 2 数据可知, 各种取代的 5-甲氧基菲啶-6(5H)䣶与苯基溴化镁和 4-氯苯基溴化镁、4-甲基苯基溴化镁 反应都可以主要得到目标化合物 3 (TLC 显示仍有痕量 的化合物 4 生成), 但菲啶酮苯环上取代基的性质和位 置对反应有一定影响(Entries $1 \sim 7,10 \sim 11$ ). 当取代基 $\mathrm{R}^{1}$ 为吸电子基团时, 表现出较低的反应活性, 而取代基 $\mathrm{R}^{1}$ 为供电子基团时则表现出较高的反应活性. 例如，当 $\mathrm{R}^{1}$ 为甲氧基时，得到的菲啶化合物 3d 的产率可达 $90 \%$ (Entry 4). 当 $\mathrm{R}^{1}$ 为甲基时, 它在菲啶酮的 9 位比在 8 位 上获得的产物产率高(Entries 2 3). 
表 2 5-甲氧基菲啶-6(5H)-酮与芳基和噻吩格氏试剂的反应 结果 $a$

Table 2 Reaction of 5-methoxyphenanthridin-6(5H)-ones with aryl and 2-thienyl magnesium bromide

\begin{tabular}{|c|c|c|c|c|c|}
\hline Entry & $\mathrm{R}^{1}$ & $\mathrm{R}^{2}$ & $\mathrm{R}^{3}$ & Product & $\begin{array}{l}\text { Isolated } \\
\text { yield } / \%\end{array}$ \\
\hline 1 & $\mathrm{H}$ & $\mathrm{CH}_{3}$ & $\mathrm{Ph}$ & $3 \mathbf{a}$ & 75 \\
\hline 2 & $8-\mathrm{CH}_{3}$ & $\mathrm{CH}_{3}$ & $\mathrm{Ph}$ & $3 \mathbf{b}$ & 62 \\
\hline 3 & 9- $\mathrm{CH}_{3}$ & $\mathrm{CH}_{3}$ & $\mathrm{Ph}$ & $3 c$ & 76 \\
\hline 4 & $9-\mathrm{OCH}_{3}$ & $\mathrm{CH}_{3}$ & $\mathrm{Ph}$ & $3 d$ & 90 \\
\hline 5 & $\mathrm{H}$ & $\mathrm{H}$ & $\mathrm{Ph}$ & $3 e$ & 83 \\
\hline 6 & $9-\mathrm{Cl}$ & $\mathrm{CH}_{3}$ & $\mathrm{Ph}$ & $3 f$ & 54 \\
\hline 7 & $9-\mathrm{F}$ & $\mathrm{CH}_{3}$ & $\mathrm{Ph}$ & $3 g$ & 45 \\
\hline 8 & $9-\mathrm{Cl}$ & $\mathrm{CH}_{3}$ & 2-Thienyl & $3 h$ & 38 \\
\hline 9 & $\mathrm{H}$ & $\mathrm{H}$ & 2-Thienyl & $3 \mathbf{i}$ & 50 \\
\hline 10 & 9- $\mathrm{OCH}_{3}$ & $\mathrm{CH}_{3}$ & $4-\mathrm{ClC}_{6} \mathrm{H}_{4}$ & $3 \mathbf{j}$ & 56 \\
\hline 11 & 9- $\mathrm{CH}_{3}$ & $\mathrm{CH}_{3}$ & 4- $\mathrm{CH}_{3} \mathrm{C}_{6} \mathrm{H}_{4}$ & $3 \mathbf{k}$ & 67 \\
\hline
\end{tabular}

${ }^{a}$ Reaction conditions: $1(0.04 \mathrm{mmol})$, aryl bromide $(0.2 \mathrm{mmol})$, magnesium (0.2 mmol), THF (4 mL), 12 14 h.

另外, 5-甲氧基菲啶-6(5H)-酮与噻吩澳化镁试剂反 应也可以顺利得到菲啶类化合物, 但是反应产率明显降 低(Entries 8９).

在研究中我们还发现, 当 5-甲氧基菲啶-6(5H)-酮化 合物与脂肪族溴代烃制成的格氏试剂，如乙基溴化镁、 正丁基澳化镁、异丁基溴化镁在上述优化反应条件下反 应时, 得到的主产物只是把 5-甲氧基菲啶酮的氮原子上 的甲氧基脱去后的菲啶酮化合物 4, 格氏试剂中的烃基 并没有出现在产物结构中(Eq. 2), 反应结果见表 3. 通 过 TLC 检测可知有痕量的化合物 3 生成.<smiles>[R1]c1ccc2c(=O)n(OC)c3ccc([R])cc3c2c1</smiles>

1

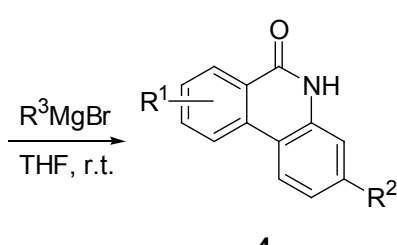

4
$\mathrm{R}^{3}=\mathrm{CH}_{3} \mathrm{CH}_{2}, n-\mathrm{Bu}, i-\mathrm{Bu}$

表 35 -甲氧基菲啶-6 $(5 H)$-酮与烷基溴化镁的反应结果 ${ }^{a}$

Table 3 Reaction of 5-methoxyphenanthridin-6(5H)-ones with alkyl magnesium bromide

\begin{tabular}{clllcc}
\hline Entry & \multicolumn{1}{c}{$\mathrm{R}^{1}$} & $\mathrm{R}^{2}$ & \multicolumn{1}{c}{$\mathrm{R}^{3}$} & Product & $\begin{array}{c}\text { Isolated } \\
\text { yield/\% }\end{array}$ \\
\hline 1 & $\mathrm{H}$ & $\mathrm{CH}_{3}$ & $i$ - $\mathrm{Bu}$ & $\mathbf{4 a}$ & 73 \\
2 & $8-\mathrm{CH}_{3}$ & $\mathrm{CH}_{3}$ & $i-\mathrm{Bu}$ & $\mathbf{4 b}$ & 81 \\
3 & $9-\mathrm{CH}_{3}$ & $\mathrm{CH}_{3}$ & $i-\mathrm{Bu}$ & $\mathbf{4 c}$ & 80 \\
4 & $\mathrm{H}$ & $\mathrm{H}$ & $i-\mathrm{Bu}$ & $\mathbf{4 d}$ & 65 \\
5 & $9-\mathrm{Cl}$ & $\mathrm{CH}_{3}$ & $i-\mathrm{Bu}$ & $\mathbf{4 e}$ & 56 \\
6 & $9-\mathrm{F}$ & $\mathrm{CH}_{3}$ & $i-\mathrm{Bu}$ & $\mathbf{4 f}$ & 52 \\
7 & $9-\mathrm{CF}_{3}$ & $\mathrm{CH}_{3}$ & $i-\mathrm{Bu}$ & $\mathbf{4 g}$ & 43 \\
8 & $8-\mathrm{CH}_{3}$ & $\mathrm{CH}_{3}$ & $\mathrm{CH} \mathrm{CH}_{2}$ & $\mathbf{4 b}$ & 42 \\
9 & $8-\mathrm{CH}_{3}$ & $\mathrm{CH}_{3}$ & $n-\mathrm{Bu}$ & $\mathbf{4 b}$ & 53 \\
\hline
\end{tabular}

${ }^{a}$ Reaction conditions: $1(0.04 \mathrm{mmol})$, alkyl bromide $(0.2 \mathrm{mmol})$, magnesium (0.2 mmol), THF (4 mL), 12 14 h.
根据文献[14]可知，Weinreb 酰胺在强碱作用下，可 发生 $\mathrm{E} 2$ 消除转变为 $N$-甲基酰胺. 当用烷基格氏试剂与 5-甲氧基菲啶- $6(5 H)$-酮化合物反应时，得到的化合物 4 正是化合物 1 发生消除反应后的产物. 这是因为烷基格 氏试剂的负电荷比苯环及杂环的格氏试剂的负电荷更 集中, 不利于和化合物 $\mathbf{4}$ 的负离子进一步反应得到化合 物 3.

\section{2 结论}

本文利用 5-甲氧基菲啶-6 $(5 H)$-酮及其衍生物和芳 基格氏试剂反应，合成了一系列菲啶衍生物，并对反应 条件进行了优化笁选，得到了最佳的反应条件. 该方法 条件温和、操作简单, 为合成菲啶衍生物提供了一种新 方法. 但是当用脂肪族格氏试剂反应时，只得到了将原 料分子中甲氧基脱去后的菲啶酮类化合物.

\section{3 实验部分}

\section{1 仪器与试剂}

红外光谱仪为 Alpha Centauri FI-IR 型仪器, 固体采 用 $\mathrm{KBr}$ 压片法, 液体采用液膜法; 核磁共振谱用 BRUKER PT jxf790425AM $400 \mathrm{MHz}$ 型核磁共振仪测 定, 以気代氯仿作为溶剂, TMS 为内标; 质谱用 QP-1000A GC-MS 测定, EI 源; 高分辨质谱用 Bruker APEX II 傅里叶变换离子回旋共振质谱仪测定, ESI 源; 熔点测定用显微熔点测定仪测定, 温度未校正. 化合物 1a 1i 按照文献[15]. 方法自制, 其它试剂均为国产分 析纯级.

\section{2 化合物 $3 a \sim 3 i$ 的合成}

于一 $50 \mathrm{~mL}$ 干燥两口瓶中, 加入镁屑 $(0.2 \mathrm{mmol})$, 氮气置换瓶中空气后, 再加入 $0.5 \mathrm{~mL}$ 干燥 $\mathrm{THF}$ 溶液, 摚 拌. 将澳苯(或 2-溴噻吩) $(0.2 \mathrm{mmol}$ )先用针管滴入 0.06 $\mathrm{mL}$, 用电吹风加热, 并使混合液保持微沸 $2 \mathrm{~min}$. 再将 剩余溴苯(或 2-溴寚吩)用 THF 稀释后，用针管缓慢滴入 至反应瓶，摚拌直至镁屑反应完全. 然后，将 3-甲基-5甲氧基菲啶-6(5H)-酮(1a) $(0.04 \mathrm{mmol})$ 的 THF 溶液, 缓 慢滴入上述反应体系中, 室温搅拌. TLC 检测反应完全 后, 向反应混合物中加入 $5 \mathrm{~mL}$ 饱和氯化铵溶液, 搅拌 $10 \mathrm{~min}$, 用乙酸乙酯 $(10 \mathrm{~mL} \times 3)$ 萃取, 分出有机相, 用 无水硫酸镁干燥，蒸去溶剂后，进行柱层析 $[V$ (石油 醚)： $V($ 乙酸乙酯 $)=15 ： 1$ ]分离得产物 $\mathbf{3 a}$.

3-甲基-6-苯基菲啶(3a)：黄色固体，产率 75\%. m.p. $103 \sim 105{ }^{\circ} \mathrm{C}$ (文献值 ${ }^{[16]} 107 \sim 108{ }^{\circ} \mathrm{C}$ ); ${ }^{1} \mathrm{H}$ NMR (400 $\left.\mathrm{MHz}, \mathrm{CDCl}_{3}\right) \delta: 8.66(\mathrm{~d}, J=8.4 \mathrm{~Hz}, 1 \mathrm{H}), 8.50(\mathrm{~d}, J=8.4$ $\mathrm{Hz}, 1 \mathrm{H}), 8.10 \sim 8.02(\mathrm{~m}, 2 \mathrm{H}), 7.83(\mathrm{t}, J=7.6 \mathrm{~Hz}, 1 \mathrm{H}), 7.73$ $(\mathrm{dd}, J=8.0,1.6 \mathrm{~Hz}, 2 \mathrm{H}), 7.60 \sim 7.48(\mathrm{~m}, 5 \mathrm{H}), 2.60(\mathrm{~s}, 3 \mathrm{H})$; 
${ }^{13} \mathrm{C}$ NMR $\left(101 \mathrm{MHz}, \mathrm{CDCl}_{3}\right) \delta: 161.2,143.9,139.9,139.0$, $130.4,129.9,129.7,128.8,128.6,128.6,128.4,126.6$, 124.9, 122.0, 121.7, 121.4, 21.6; IR (KBr) v: 3064, 3023, 2923, 2853, 1616, 1563, 1481, 1450, $1362 \mathrm{~cm}^{-1}$; MS (70 eV) $m / z(\%): 270\left(\mathrm{M}^{+}+1,9\right), 269$ (50), 268 (100), 133 (14), 71 (17), 57 (23).

3,8-二甲基-6-苯基菲啶 (3b)：淡黄色液体，产率 $62 \%$. ${ }^{1} \mathrm{H}$ NMR (400 MHz, $\left.\mathrm{CDCl}_{3}\right) \delta: 8.50(\mathrm{~d}, J=8.0 \mathrm{~Hz}$, $1 \mathrm{H}), 8.45 \sim 8.37(\mathrm{~m}, 1 \mathrm{H}), 8.02(\mathrm{~s}, 1 \mathrm{H}), 7.82(\mathrm{~s}, 1 \mathrm{H}), 7.71$ (d, $J=7.2 \mathrm{~Hz}, 2 \mathrm{H}), 7.66 \sim 7.40$ (m, 5H), 2.57 (s, 3H), 2.47 (s, 3H); ${ }^{13} \mathrm{C}$ NMR $\left(101 \mathrm{MHz}, \mathrm{CDCl}_{3}\right) \delta: 160.9,143.6$, $140.1,138.4,136.5,132.1,129.8,129.7,128.5,128.5$, $128.3,128.2,128.1,125.0,121.9,121.5,21.6,21.5$; IR (KBr) $v$ : 3056, 3026, 2920, 2858, 1620, 1560, 1479, 1449, $1363 \mathrm{~cm}^{-1}$; HRMS calcd for $\mathrm{C}_{21} \mathrm{H}_{18} \mathrm{~N}\left(\mathrm{M}^{+}+1\right) 284.1434$, found 284.1439 .

3,9-二甲基-6-苯基菲啶 (3c)：淡黄色液体，产率 76\%. ${ }^{1} \mathrm{H}$ NMR (400 MHz, $\left.\mathrm{CDCl}_{3}\right) \delta: 8.40$ (s, 1H), 8.29 (s, $1 \mathrm{H}), 7.98(\mathrm{~s}, 1 \mathrm{H}), 7.93(\mathrm{~d}, J=8.4 \mathrm{~Hz}, 1 \mathrm{H}), 7.73 \sim 7.68(\mathrm{~m}$, 2H), $7.56 \sim 7.44(\mathrm{~m}, 4 \mathrm{H}), 7.34(\mathrm{~d}, J=7.2 \mathrm{~Hz}, 1 \mathrm{H}), 2.52$ (s, $3 \mathrm{H}), 2.47(\mathrm{~s}, 3 \mathrm{H}) ;{ }^{13} \mathrm{C}$ NMR (101 MHz, CDCl3) $\delta: 160.0$, $142.8,140.5,140.1,138.2,136.0,133.3,130.1,129.7$, 128.6, 128.3, 128.2, 128.1, 123.1, 121.9, 121.5, 20.3, 20.0; IR (KBr) v: 3058, 3026, 2921, 2860, 1618, 1563, 1493, 1450, $1363 \mathrm{~cm}^{-1}$; HRMS calcd for $\mathrm{C}_{21} \mathrm{H}_{18} \mathrm{~N}\left(\mathrm{M}^{+}+1\right)$ 284.1434, found 284.1432.

3-甲基-6-苯基-9-甲氧基菲啶(3d): 无色液体, 产率 90\%. ${ }^{1} \mathrm{H}$ NMR (400 MHz, $\left.\mathrm{CDCl}_{3}\right) \delta: 8.39(\mathrm{~d}, J=8.0 \mathrm{~Hz}$, 1H), 7.97 (dd, $J=18.8,9.6 \mathrm{~Hz}, 3 \mathrm{H}), 7.70(\mathrm{~d}, J=6.4 \mathrm{~Hz}$, $2 \mathrm{H}), 7.55 \sim 7.44(\mathrm{~m}, 4 \mathrm{H}), 7.15(\mathrm{~d}, J=9.0 \mathrm{~Hz}, 1 \mathrm{H}), 4.03$ (d, $J=1.6 \mathrm{~Hz}, 3 \mathrm{H}), 2.58(\mathrm{~s}, 3 \mathrm{H}) ;{ }^{13} \mathrm{C} \mathrm{NMR}\left(101 \mathrm{MHz}, \mathrm{CDCl}_{3}\right)$ $\delta: 161.2,144.3,140.0,139.0,135.6,130.8,129.8,129.6$, $128.5,128.3,128.1,121.7,121.2,119.9,116.8,102.6$, 55.5, 21.5; IR (KBr) v: 3054, 3014, 2925, 2853, 1614, $1577,1496,1456,1372 \mathrm{~cm}^{-1}$; HRMS calcd for $\mathrm{C}_{21} \mathrm{H}_{18} \mathrm{NO}$ $\left(\mathrm{M}^{+}+1\right) 300.1383$, found 300.1386 .

6-苯基菲啶(3e)：白色固体，产率 83\%. m.p. 102 $104{ }^{\circ} \mathrm{C}$ (文献值 $\left.{ }^{[16]} 103.5 \sim 104.5{ }^{\circ} \mathrm{C}\right) ;{ }^{1} \mathrm{H}$ NMR $(400 \mathrm{MHz}$, $\left.\mathrm{CDCl}_{3}\right) \delta: 8.62(\mathrm{~d}, J=8.3 \mathrm{~Hz}, 1 \mathrm{H}), 8.54(\mathrm{~d}, J=7.0 \mathrm{~Hz}$, $1 \mathrm{H}), 8.19(\mathrm{~d}, J=8.2 \mathrm{~Hz}, 1 \mathrm{H}), 8.04(\mathrm{~d}, J=8.2 \mathrm{~Hz}, 1 \mathrm{H}), 7.78$ (t, $J=7.6 \mathrm{~Hz}, 1 \mathrm{H}), 7.73 \sim 7.66(\mathrm{~m}, 3 \mathrm{H}), 7.65 \sim 7.59(\mathrm{~m}$, $1 \mathrm{H}), 7.57 \sim 7.43(\mathrm{~m}, 4 \mathrm{H}) ;{ }^{13} \mathrm{C} \mathrm{NMR}\left(101 \mathrm{MHz}, \mathrm{CDCl}_{3}\right) \delta$ : $161.2,143.8,139.8,133.4,130.5,130.3,129.7,128.9$, $128.8,128.7,128.4,127.1,126.9,125.2,123.7,122.1$, 121.9; IR (KBr) v: 3066, 3014, 2925, 2852, 1611, 1562,
1522, 1456, $1359 \mathrm{~cm}^{-1}$; MS (70 eV) m/z (\%): $256\left(\mathrm{M}^{+}+1\right.$, 9), 255 (50), 254 (100), 253(19), 127 (12), 44 (3).

3-甲基-6-苯基-9-氯菲啶 (3f): 淡黄色固体, 产率 $54 \%$. m.p. $117 \sim 119{ }^{\circ} \mathrm{C} ;{ }^{1} \mathrm{H}$ NMR $\left(400 \mathrm{MHz}, \mathrm{CDCl}_{3}\right) \delta$ : $8.56(\mathrm{~d}, J=1.6 \mathrm{~Hz}, 1 \mathrm{H}), 8.20(\mathrm{~s}, 1 \mathrm{H}), 8.03 \sim 7.96(\mathrm{~m}, 2 \mathrm{H})$, $7.70 \sim 7.66(\mathrm{~m}, 2 \mathrm{H}), 7.57 \sim 7.46(\mathrm{~m}, 5 \mathrm{H}), 2.48(\mathrm{~s}, 3 \mathrm{H}) ;{ }^{13} \mathrm{C}$ NMR $\left(101 \mathrm{MHz}, \mathrm{CDCl}_{3}\right) \delta: 159.7,143.0,139.6,139.2$, $136.7,134.4,130.4,130.3,129.7,129.6,128.7,128.4$, 127.0, 123.2, 121.9, 121.6, 20.3; IR (KBr) v: 3058, 3026, 2943, 2855, 1602, 1565, 1481, 1453, $1361 \mathrm{~cm}^{-1}$; HRMS calcd for $\mathrm{C}_{20} \mathrm{H}_{15} \mathrm{ClN}\left(\mathrm{M}^{+}+1\right) 304.0888$, found 304.0886.

3-甲基-6-苯基-9-氟菲啶(3g)：白色固体，产率 45\%. m.p. $133 \sim 136{ }^{\circ} \mathrm{C} ;{ }^{1} \mathrm{HNMR}\left(400 \mathrm{MHz}, \mathrm{CDCl}_{3}\right) \delta: 8.50(\mathrm{~d}$, $J=8.8 \mathrm{~Hz}, 1 \mathrm{H}), 8.43(\mathrm{~d}, J=8.4 \mathrm{~Hz}, 1 \mathrm{H}), 8.21$ (d, $J=2.0$ $\mathrm{Hz}, 1 \mathrm{H}), 8.03$ (s, 1H), 7.90 (dd, $J=8.8,2.0 \mathrm{~Hz}, 1 \mathrm{H}), 7.70$ (dd, $J=7.6,1.6 \mathrm{~Hz}, 2 \mathrm{H}), 7.61 \sim 7.48$ (m, 4H), 2.59 (s, 3H); ${ }^{13} \mathrm{C} \mathrm{NMR}\left(101 \mathrm{MHz}, \mathrm{CDCl}_{3}\right) \delta: 160.0,143.8,139.5,139.2$, 133.6, 132.2, 131.0, 130.0, 129.6, 129.1, 128.9, 128.5, 126.2, 123.9, 121.5, 120.8, 120.6, 109.7, 21.5; IR (KBr) v: 3052, 3018, 2925, 2853, 1619, 1581, 1492, 1458, 1366 $\mathrm{cm}^{-1}$; HRMS calcd for $\mathrm{C}_{20} \mathrm{H}_{15} \mathrm{FN}\left(\mathrm{M}^{+}+1\right) 288.1183$, found 288.1187 .

3-甲基-6-(2-噻吩)-9-氯菲啶(3h): 黄色固体, 产率 38\%. m.p. $157 \sim 159{ }^{\circ} \mathrm{C} ;{ }^{1} \mathrm{H}$ NMR (400 MHz, $\left.\mathrm{CDCl}_{3}\right) \delta$ : $8.55(\mathrm{~d}, J=1.6 \mathrm{~Hz}, 1 \mathrm{H}), 8.44(\mathrm{~d}, J=8.8 \mathrm{~Hz}, 1 \mathrm{H}), 8.17$ (s, $1 \mathrm{H}), 7.95(\mathrm{~s}, 1 \mathrm{H}), 7.66 \sim 7.42(\mathrm{~m}, 4 \mathrm{H}), 7.22$ (dd, $J=5.2$, $3.6 \mathrm{~Hz}, 1 \mathrm{H}), 2.48(\mathrm{~s}, 3 \mathrm{H}) ;{ }^{13} \mathrm{C}$ NMR $\left(101 \mathrm{MHz}, \mathrm{CDCl}_{3}\right) \delta$ : $152.5,142.9,142.5,139.3,136.9,136.8,134.7,130.2$, $129.7,128.9,127.8,127.3,122.8,121.9,121.8,120.5$, 20.4; IR (KBr) v: 3072, 2922, 2857, 1601, 1558, 1481, 1431, 1361, $1235 \mathrm{~cm}^{-1}$; HRMS calcd for $\mathrm{C}_{18} \mathrm{H}_{13} \mathrm{ClNS}$ $\left(\mathrm{M}^{+}+1\right) 310.0452$, found 310.0456 .

6-(2-噻吩)-菲啶 (3i): 淡黄色固体, 产率 50\%. m.p. 92 94 ${ }^{\circ} \mathrm{C} ;{ }^{1} \mathrm{H}$ NMR $\left(400 \mathrm{MHz}, \mathrm{CDCl}_{3}\right) \delta: 8.70(\mathrm{~d}, J=8.4$ $\mathrm{Hz}, 1 \mathrm{H}), 8.58(\mathrm{dd}, J=8.0,4.0 \mathrm{~Hz}, 2 \mathrm{H}), 8.21(\mathrm{~d}, J=8.0 \mathrm{~Hz}$, $1 \mathrm{H}), 7.88(\mathrm{t}, J=7.6 \mathrm{~Hz}, 1 \mathrm{H}), 7.78 \sim 7.62(\mathrm{~m}, 4 \mathrm{H}), 7.57(\mathrm{~d}$, $J=5.2 \mathrm{~Hz}, 1 \mathrm{H}), 7.24(\mathrm{dd}, J=5.6,4.8 \mathrm{~Hz}, 1 \mathrm{H}) ;{ }^{13} \mathrm{C} \mathrm{NMR}$ $\left(101 \mathrm{MHz}, \mathrm{CDCl}_{3}\right) \delta: 154.1,143.8,142.6,133.7,130.6$, $130.3,129.2,128.9,128.1,127.9,127.4,127.3,127.0$, 124.8, 123.6, 122.4, 121.9; IR (KBr) v: 3072, 2925, 2852, $1605,1561,1481,1429,1359,1216 \mathrm{~cm}^{-1}$; HRMS calcd for $\mathrm{C}_{17} \mathrm{H}_{12} \mathrm{NS}\left(\mathrm{M}^{+}+1\right) 262.0685$, found 262.0688.

3-甲基-6-(4-氯苯)-9-甲氧基菲啶(3j): 白色固体，产 率 56\%. m.p. $161 \sim 162{ }^{\circ} \mathrm{C} ;{ }^{1} \mathrm{H}$ NMR $\left(400 \mathrm{MHz}, \mathrm{CDCl}_{3}\right)$ $\delta: 8.39$ (d, $J=8.6 \mathrm{~Hz}, 1 \mathrm{H}), 7.94$ (t, $J=12.6 \mathrm{~Hz}, 3 \mathrm{H}), 7.65$ 
(d, $J=8.0 \mathrm{~Hz}, 2 \mathrm{H}), 7.49$ (dd, $J=15.2,8.4 \mathrm{~Hz}, 3 \mathrm{H}), 7.16$ $(\mathrm{d}, J=9.0 \mathrm{~Hz}, 1 \mathrm{H}), 4.03(\mathrm{~s}, 3 \mathrm{H}), 2.58(\mathrm{~s}, 3 \mathrm{H}) ;{ }^{13} \mathrm{C} \mathrm{NMR}$ $\left(101 \mathrm{MHz}, \mathrm{CDCl}_{3}\right) \delta: 161.3,159.4,144.3,139.2,138.5$, $135.7,134.7,131.1,130.3,129.8,128.5,128.3,121.7$, $121.3,119.7,116.9,102.7,55.5,21.5$; IR (KBr) v: 3058, 2920, 2860, 1610, 1558, 1490, 1452, 1361, $1226 \mathrm{~cm}^{-1}$; HRMS calcd for $\mathrm{C}_{21} \mathrm{H}_{17} \mathrm{ClNO}\left(\mathrm{M}^{+}+1\right)$ 334.0993, found 334.0995 .

3,9-二甲基-6-(4-甲基苯)菲啶(3k)：淡黄色固体，产 率 67\%. m.p. 85 86 ${ }^{\circ} \mathrm{C} ;{ }^{1} \mathrm{H}$ NMR (400 MHz, $\left.\mathrm{CDCl}_{3}\right) \delta$ : $8.43(\mathrm{~d}, J=8.4 \mathrm{~Hz}, 1 \mathrm{H}), 8.38(\mathrm{~s}, 1 \mathrm{H}), 8.03 \sim 7.94(\mathrm{~m}, 2 \mathrm{H})$, 7.61 (d, $J=7.6 \mathrm{~Hz}, 2 \mathrm{H}), 7.45$ (d, $J=8.4 \mathrm{~Hz}, 1 \mathrm{H}), 7.34$ (d, $J=6.8 \mathrm{~Hz}, 3 \mathrm{H}), 2.60$ (s, 3H), 2.57 (s, 3H), 2.46 (s, 3H); ${ }^{13} \mathrm{C}$ NMR (101 MHz, $\mathrm{CDCl}_{3}$ ) $\delta: 161.1,144.1,140.6,138.6$, $138.3,137.2,133.6,129.7,129.6,128.9,128.7,128.2$, 123.1, 121.6, 121.5, 121.1, 22.1, 21.5, 21.3; IR (KBr) $v$ : 3026, 2916, 2857, 1616, 1560, 1492, 1450, 1361, 1228 $\mathrm{cm}^{-1}$; HRMS calcd for $\mathrm{C}_{22} \mathrm{H}_{20} \mathrm{~N}\left(\mathrm{M}^{+}+1\right) 298.1590$, found 298.1593.

\section{3 化合物 $4 \mathrm{a} \sim 4 \mathrm{~g}$ 的合成}

于一 $50 \mathrm{~mL}$ 干燥两口瓶中, 加入镁屑 $(0.2 \mathrm{mmol})$, 一小粒碘, 氮气置换瓶中空气后, 加入 $0.5 \mathrm{~mL}$ 干燥 $\mathrm{THF}$ 溶液, 摚拌. 将溴代异丁烷 $(0.2 \mathrm{mmol})$ 先用针管滴入 $0.06 \mathrm{~mL}$, 用电吹风加热反应瓶, 溶液由黄色变为无色. 再将剩余溴代异丁烷用 THF 稀释后, 用针管缓慢滴入 反应瓶. 搅拌至镁屑基本消失. 然后, 将 3-甲基-5-甲氧 基菲啶-6(5H)-酮(1a) (0.04 mmol)的 THF 溶液缓慢滴入 上述反应体系中, 室温搅拌. TLC 检测反应完全后, 向 反应混合物中加入 $5 \mathrm{~mL}$ 饱和氯化铵溶液, 搅拌 $10 \mathrm{~min}$, 用乙酸乙酯 $(10 \mathrm{~mL} \times 3)$ 萃取, 分出有机相, 用无水硫酸 镁干燥, 蒸去溶剂后, 进行柱层析 $[V$ (石油醚)： $V$ (乙酸 乙酯) $=2: 1$ ], 分离得产物 $4 \mathbf{a}$.

3-甲基菲啶-6(5H)-酩(4a) ${ }^{[17]}$ : 白色固体，产率 73\%. m.p. $>250{ }^{\circ} \mathrm{C} ;{ }^{1} \mathrm{H}$ NMR (400 MHz, DMSO- $\left.d_{6}\right) \delta: 11.63$ $(\mathrm{s}, 1 \mathrm{H}), 8.45(\mathrm{~d}, J=8.0 \mathrm{~Hz}, 1 \mathrm{H}), 8.34 \sim 8.30(\mathrm{~m}, 1 \mathrm{H}), 8.26$ $(\mathrm{d}, J=8.0 \mathrm{~Hz}, 1 \mathrm{H}), 7.89 \sim 7.77(\mathrm{~m}, 1 \mathrm{H}), 7.61(\mathrm{t}, J=7.6$ $\mathrm{Hz}, 1 \mathrm{H}), 7.09$ (d, $J=8.0 \mathrm{~Hz}, 1 \mathrm{H}), 2.39$ (s, 3H); ${ }^{13} \mathrm{C}$ NMR (101 MHz, DMSO) $\delta$ : 160.9, 139.3, 136.6, 134.3, 132.7, $127.4,125.3,123.5,123.1,122.3,115.9,115.2,21.1$; IR (KBr) v: 3026, 1662, $1363 \mathrm{~cm}^{-1}$; MS (70 eV) m/z (\%): 210 $\left(\mathrm{M}^{+}+1,15\right), 209$ (100), 208 (47), 180 (16), 152 (10),76 (5).

3,8-二甲基菲啶-6 $(5 H)$-酮(4b) ${ }^{[18]}$ : 白色固体, 产率 $81 \%$. m.p. $>250{ }^{\circ} \mathrm{C} ;{ }^{1} \mathrm{H}$ NMR (400 MHz, DMSO-d $\left.d_{6}\right) \delta$ : $11.56(\mathrm{~s}, 1 \mathrm{H}), 8.32$ (d, $J=8.0 \mathrm{~Hz}, 1 \mathrm{H}), 8.20(\mathrm{~d}, J=8.0 \mathrm{~Hz}$,
$1 \mathrm{H}), 8.11(\mathrm{~s}, 1 \mathrm{H}), 7.64(\mathrm{~d}, J=8.0 \mathrm{~Hz}, 1 \mathrm{H}), 7.15(\mathrm{~s}, 1 \mathrm{H})$, $7.06(\mathrm{~d}, J=8.0 \mathrm{~Hz}, 1 \mathrm{H}), 2.47(\mathrm{~s}, 3 \mathrm{H}), 2.38(\mathrm{~s}, 3 \mathrm{H}) ;{ }^{13} \mathrm{C}$ NMR (101 MHz, DMSO- $\left.d_{6}\right) \delta: 160.9,138.8,137.0,136.2$, $133.8,131.9,127.1,125.2,123.4,122.8,122.3,115.8$, 115.3, 21.1, 20.9; IR (KBr) v: 3026, 1667, $1360 \mathrm{~cm}^{-1}$; MS (70 eV) m/z (\%): $224\left(\mathrm{M}^{+}+1,8\right), 223$ (37), 222 (15), 149 (13), 194 (3), 57 (100), 44 (68).

3,9-二甲基菲啶-6(5H)-酩(4c) ${ }^{[18]}$ : 白色固体，产率 $80 \%$. m.p. $>250{ }^{\circ} \mathrm{C} ;{ }^{1} \mathrm{H}$ NMR $\left(400 \mathrm{MHz}, \mathrm{DMSO}-d_{6}\right) \delta$ : 11.52 (s, 1H), 8.21 (dd, $J=19.2,9.6 \mathrm{~Hz}, 4 \mathrm{H}), 7.41$ (d, $J=$ $8.0 \mathrm{~Hz}, 1 \mathrm{H}), 7.15(\mathrm{~s}, 1 \mathrm{H}), 7.07$ (d, $J=8.0 \mathrm{~Hz}, 1 \mathrm{H}), 2.52$ (s, $3 \mathrm{H}), 2.38(\mathrm{~s}, 3 \mathrm{H}) ;{ }^{13} \mathrm{C}$ NMR (101 MHz, DMSO- $\left.d_{6}\right) \delta$ : $160.9,142.8,139.2,136.7,134.3,128.6,127.4,123.3$, 123.0, 122.2, 115.9, 115.2, 21.5, 21; IR (KBr) v: 3027, 1666, $1363 \mathrm{~cm}^{-1}$; MS (70 eV) $\mathrm{m} / z(\%): 224\left(\mathrm{M}^{+}+1,16\right)$, 223 (100), 222 (37), 194 (9), 149 (5), 57 (27), 44 (65).

菲啶-6 $(5 H)$-酮(4d): 白色固体，产率 $65 \%$. m.p. $>250{ }^{\circ} \mathrm{C}$ (文献值 ${ }^{[17]} 308 \sim 310{ }^{\circ} \mathrm{C}$ ); ${ }^{1} \mathrm{H}$ NMR $(400 \mathrm{MHz}$, DMSO- $\left.d_{6}\right) \delta: 11.72(\mathrm{~s}, 1 \mathrm{H}), 8.52(\mathrm{~d}, J=8.0 \mathrm{~Hz}, 1 \mathrm{H}), 8.38$ (dd, $J=18.8,7.6 \mathrm{~Hz}, 2 \mathrm{H}), 7.87$ (dd, $J=11.2,4.0 \mathrm{~Hz}, 1 \mathrm{H})$, $7.66(\mathrm{t}, J=7.6 \mathrm{~Hz}, 1 \mathrm{H}), 7.51(\mathrm{t}, J=7.2 \mathrm{~Hz}, 1 \mathrm{H}), 7.40$ (d, $J=8.0 \mathrm{~Hz}, 1 \mathrm{H}), 7.28(\mathrm{t}, J=7.6 \mathrm{~Hz}, 1 \mathrm{H}) ;{ }^{13} \mathrm{C} \mathrm{NMR}(101$ MHz, DMSO- $\left.d_{6}\right) \delta: 160.7,136.5,134.2,132.7,129.5$, $127.8,127.4,125.6,123.2,122.5,122.2,117.5,116.1$; IR (KBr) v: 3043, 1662, $1366 \mathrm{~cm}^{-1}$; MS (70 eV) m/z (\%): 196 $\left(\mathrm{M}^{+}+1,14\right), 195$ (100), 194 (10), 57 (6), 44 (65).

3-甲基-9-氯菲啶-6(5H)-酮 $(4 e)^{[18]}$ : 白色固体，产率 $56 \%$. m.p. $>250{ }^{\circ} \mathrm{C} ;{ }^{1} \mathrm{H}$ NMR (400 MHz, DMSO- $\left.d_{6}\right) \delta$ : $11.69(\mathrm{~s}, 1 \mathrm{H}), 8.52$ (s, 1H), 8.28 (d, J=8.0 Hz, 2H), 7.62 $(\mathrm{d}, J=8.0 \mathrm{~Hz}, 1 \mathrm{H}), 7.15$ (s, 1H), 7.08 (d, $J=8.0 \mathrm{~Hz}, 1 \mathrm{H})$, $2.39(\mathrm{~s}, 3 \mathrm{H}) ;{ }^{13} \mathrm{C}$ NMR (101 MHz, DMSO- $\left.d_{6}\right) \delta: 160.2$, 140.2 , 138.1, 137.0, 136.1, 129.6, 127.4, 127.3, 123.9, 123.6, 122.0, 115.9, 114.2, 21.1; IR (KBr) v: 3027, 1668, $1360 \mathrm{~cm}^{-1}$; MS (70 eV) $\mathrm{m} / z(\%): 245\left(\mathrm{M}^{+}+1,33\right), 244$ (28), 243 (100), 209 (17), 180 (10), 76 (7).

3-甲基-9-氟菲啶-6 $(5 H)$-酮(4f)：白色固体，产率 $52 \%$. m.p. $>250{ }^{\circ} \mathrm{C} ;{ }^{1} \mathrm{H}$ NMR (400 MHz, DMSO- $\left.d_{6}\right) \delta$ : 8.39 (d, $J=8.0 \mathrm{~Hz}, 1 \mathrm{H}), 8.20(\mathrm{~d}, J=8.0 \mathrm{~Hz}, 1 \mathrm{H}), 7.79$ (t, $J=7.6 \mathrm{~Hz}, 1 \mathrm{H}), 7.57$ (t, $J=7.6 \mathrm{~Hz}, 1 \mathrm{H}), 7.13$ (s, 1H), 7.04 $(\mathrm{d}, J=7.6 \mathrm{~Hz}, 1 \mathrm{H}), 2.35(\mathrm{~s}, 3 \mathrm{H}) ;{ }^{13} \mathrm{C} \mathrm{NMR}(101 \mathrm{MHz}$, DMSO- $\left.d_{6}\right) \delta: 160.9,139.3,136.6,134.3,132.6,130.8$, $130.7,127.4,125.3,123.7,123.4,123.1,122.3,115.9$, 21.1; IR (KBr) v: 3024, 1663, $1361 \mathrm{~cm}^{-1}$; HRMS calcd for $\mathrm{C}_{14} \mathrm{H}_{11} \mathrm{FNO}\left(\mathrm{M}^{+}+1\right) 228.0819$, found 228.0823.

3-甲基-9-三氟甲基菲啶-6(5H)-酮(4g)：白色固体， 
产率 43\%. m.p. $>250{ }^{\circ} \mathrm{C} ;{ }^{1} \mathrm{H}$ NMR (400 MHz, DMSO- $\left.d_{6}\right)$ $\delta: 11.83(\mathrm{~s}, 1 \mathrm{H}), 8.74(\mathrm{~s}, 1 \mathrm{H}), 8.41(\mathrm{dd}, J=25.2,8.0 \mathrm{~Hz}$, 2H), $7.86(\mathrm{~d}, J=7.8 \mathrm{~Hz}, 1 \mathrm{H}), 7.20 \sim 7.01(\mathrm{~m}, 2 \mathrm{H}), 2.37(\mathrm{~s}$, $3 \mathrm{H}) ;{ }^{13} \mathrm{C}$ NMR (101 MHz, DMSO-d $) \delta: 160.0,140,5$, $136.9,134.9,132.8,132.5,131.0,128.9,127.9,123.9$, $123.8,123.7,123.2,119.8,116.0,114.4,21.1$; IR (KBr) $v$ : $3028,1667,1361 \mathrm{~cm}^{-1}$; HRMS calcd for $\mathrm{C}_{15} \mathrm{H}_{11} \mathrm{~F}_{3} \mathrm{NO}$ $\left(\mathrm{M}^{+}+1\right) 278.0787$, found 278.0790.

辅助材料(Supporting Information) 合成产物的核磁 共振氢谱、碳谱和高分辨质谱. 这些材料可以免费从本 刊网站(http://sioc-journal.cn/)上下载.

\section{References}

[1] (a) Viladomat, F.; Selles, M.; Codina, C.; Bastida, J. Planta Med. 1997, 63, 583.

(b) Ali, A. A.; El Sayed, H. M.; Abdallah, O. M.; Steglich, W. Phytochemistry 1986, 25, 2399.

(c) Krane, B. D.; Fagbule, M. O.; Shamma, M. J. Nat. Prod. 1984, 47,1 .

[2] (a) Bernardo, P. H.; Wan, K. F.; Sivaraman, T.; Xu, J.; Moore, F. K.; Hung, A. W.; Mok, H. Y. K.; Yu, V. C.; Chai, C. L. L. J. Med. Chem. 2008, 51, 6699.

(b) Zhu, S.; Ruchelman, A. L.; Zhou, N.; Liu, A.; Liu, L. F.; LaVoie, E. J. Bioorg. Med.Chem. 2005, 13, 6782.

(c) Lewis, W. G.; Green, L. G.; Grynszpan, F.; Radic, Z.; Carlier, P. R.; Taylor, P.; Finn,M. G.; Sharpless, K. B. Angew. Chem., Int. Ed. 2002, 41, 1053.

(d) Lynch, M. A.; Duval, O.; Sukhanova, A.; Devy, J.; MacKay, S. P.; Waigh, R. D.; Nabiev, I. Bioorg. Med. Chem. Lett. 2001, 11, 2643.

[3] (a) Stevens, N.; O'Connor, N.; Vishwasrao, H.; Samaroo, D.; Kandel, E. R.; Akins, D. L.; Drain, C. M.; Turro, N. J. J. Am. Chem. Soc. 2008, 130, 7182 .

(b) Bondarev, S. L.; Knyukshto, V. N.; Tikhomirov, S. A.; Pyrko, A. N. Opt. Spectrosc. 2006, 100, 386.

(c) Zhang, J.; Lakowicz, J. R. J. Phys. Chem. B 2005, 109, 8701.

[4] (a) Krane, B. D.; Fagbule, M. O.; Shamma, M. J. Nat. Prod. 1984,
$47,1$.

(b) Nakanishi, T.; Suzuki, M.; Mashiba, A.; Ishikawa, K.; Yokotsuka, T. J. Org. Chem. 1998, 63, 4235.

[5] (a) Alonso, R.; Campos, P. J.; García, B.; Rodríguez, M. A. Org. Lett. 2006, 8, 3521.

(b) Moreno, I.; Tellitu, I.; Etayo, J.; SanMartín, R.; Domínguez, E. Tetrahedron. 2001, 57, 5403.

[6] (a) Shou, W. G.; Yang, Y. Y.; Wang, Y. G. J. Org. Chem. 2006, 71, 9241.

(b) Pawlas, J.; Begtrup, M. Org. Lett. 2002, 4, 2687.

(c) Sanz, R.; Fernández, Y.; Castroviejo, M. P.; Pérez, A.; Fañanás, F. J. Eur. J. Org. Chem. 2007, 62.

(d) Nakanishi, T.; Suzuki, M. Org. Lett. 1999, 1, 985.

[7] Shabashov, D.; Daugulis, O. J. Org. Chem. 2007, 72, 7720.

[8] Linsenmeier, A. M.; Williams, C. M.; Bräse, S. J. Org. Chem. 2011, 76, 9127.

[9] Read, M. L.; Gundersen, L. L. J. Org. Chem. 2013, 78, 1311.

[10] (a) Deb, I.; Yoshikai, N. Org. Lett. 2013, 15, 4254.

(b) Zhang, L.; Ang, G. Y.; Chiba, S.; Org. Lett. 2010, 12, 3682.

(c) Wang, W.Y.; Feng, X.; Hu, B. L.; Deng, C. L.; Zhang, Y. G. J. Org. Chem. 2013, 78, 6205.

(d) Gerfaud, T.; Neuville, L.; Zhu, J. P. Angew. Chem. 2009, 121, 580 .

[11] Nahm, S.; Weinreb, S. M. Tetrahedron Lett. 1981, 22, 3815.

[12] (a) Borrero, N. V.; Aponick, A. J. Org. Chem. 2012, 77, 8410.

(b) Davis. F. A.; Xu, P. J. Org. Chem. 2011, 76, 3329.

(c) Paek, S. M.; Seo, S. Y.; Kim, S. H.; Suh, Y. G. Org. Lett. 2005, 7,3159.

[13] (a) Niu, T.; Zhang, W. M.; Huang, D. F.; Xu, C. M.; Wang, H. F.; Hu, Y. L. Org. Lett. 2009, 11, 4474.

(b) Quan, F.; Huang, D. F.; Xu, C. M.; Niu, T.; Hu, Y. L. Chin. J. Org. Chem. 2009, 29, 450 (in Chinese).

(权锋, 黄丹凤, 徐长明, 牛腾, 胡雨来, 有机化学, 2009, 29, 450.)

[14] Graham, S. L.; Scholz, T. H. Tetrahedron Lett. 1990, 31, 6269

[15] Karthikeyan, J.; Cheng, C.-H. Angew. Chem., Int. Ed. 2011, 50, 9880.

[16] Peng, J. S.; Chen, T. H.; Chen, C. X.; Li, B. J. Org. Chem. 2011, 76, 9507.

[17] Bhakuni, B. S.; Kumar, A.; Balkrishna, S. J.; Sheikh, J. A.; Konar, S.; Kumar, S. Org. Lett. 2012, 14, 2838.

[18] Gui, Q. W.; Yang, Z. Y.; Chen, X.; Liu, J. D.; Tan, Z.; Guo, R. Q.; Yu, W. Synlett 2013, 1016. 